\title{
Estimating and Approximating the Total $\pi$-Electron Energy of Benzenoid Hydrocarbons
}

\author{
I. Gutman, J. H. Koolen ${ }^{\mathrm{a}}$, V. Moulton ${ }^{\mathrm{b}}$, M. Parac ${ }^{\mathrm{c}}$, T. Soldatović, and D. Vidović \\ Faculty of Science, University of Kragujevac, P. O. Box 60, YU-34000 Kragujevac, Yugoslavia \\ ${ }^{a}$ FSPM-Strukturbildungsprozesse, University of Bielefeld, D-33501 Bielefeld, Germany \\ ${ }^{\mathrm{b}}$ Physics and Mathematics Department (FMI), Mid Sweden University, \\ Sundsvall, S-85170, Sweden \\ ${ }^{c}$ Faculty of Physical Chemistry, University of Belgrade, YU-11001 Beograd, Yugoslavia \\ Reprint requests to Prof. I. G.; Fax: +381 34 335040; E-mail: gutman@ knez.uis.kg.ac.yu \\ Z. Naturforsch. 55 a, 507-512 (2000); received January 27, 2000 \\ Lower and upper bounds as well as approximate formulas for the total $\pi$-electron energy $(E)$ \\ of benzenoid hydrocarbons are deduced, depending only on the number of carbon atoms $(n)$ \\ and number of carbon-carbon bonds $(m)$. These are better than the several previously known \\ $(n, m)$-type estimates and approximations for $E$.
}

Key words: Total $\pi$-Electron Energy; Benzenoid Hydrocarbons.

\section{Introduction}

In this paper we are concerned with the total $\pi$ electron energy $(E)$ of conjugated hydrocarbons, as calculated within the tight-binding Hückel molecularorbital (HMO) model. As usual we express $E$ in the units of the HMO resonance integral $\beta$. At the very beginning we wish to remind the readers that $E$ is related not only to $\pi$-electrons, but also pertains to the (experimentally measurable) enthalpies of the underlying compounds. In particular, the heats of atomization are reproduced via [1].

$$
\Delta H_{a}=-\left[n_{\mathrm{CH}} E_{\mathrm{CH}}+m E_{\mathrm{CC}}^{0}+\beta^{\prime} E\right],
$$

where $n_{\mathrm{CH}}$ and $m$ are the numbers of carbon-hydrogen and carbon-carbon bonds, respectively, whereas the optimal values of the constants $E_{\mathrm{CH}}, E_{\mathrm{CC}}^{0}$, and $\beta^{\prime}$ are $-411.09 \mathrm{~kJ} / \mathrm{mol},-325.18 \mathrm{~kJ} / \mathrm{mol}$ and $-137.00 \mathrm{~kJ} / \mathrm{mol}$, respectively. The quantity $\beta^{\prime}$ is not just the HMO carbon-carbon resonance integral, but contains contributions coming from the compression/extension of the carbon-carbon bonds. Formula (1) is applicable to conjugated hydrocarbons the molecules of which do not deviate much from planarity and in which steric effects are relatively small. Benzenoid hydrocarbons are typical compounds satisfying these conditions. For them (1) yields enthalpies that are in surprisingly good agreement with experimental values; for details see the original article of Schaad and Hess [1], pp. 135 - 136 and $151-153$ of the book [2] or the review [3]. Recall that $n_{\mathrm{CH}}$ in (1) is equal to $3 n-2 m$.

A remarkable property of $E$ is that its gross part (well over 99\%) is determined by only two structural parameters: the number of carbon atoms $(n)$ and the number of carbon-carbon bonds $(m)$. This fact has been known for a long time (see e.g. [4, 5]), but was put on a quantitative footing by McClelland in 1971 [6]. McClelland showed that

$$
E \leq \sqrt{2 m n}
$$

and that for a suitably chosen constant $A$, the expression

$$
E \approx A \sqrt{2 m n}
$$

is a very good approximation for $E$. This especially holds for benzenoid hydrocarbons. Comparative studies $[3,7,8]$ of several dozens of $(n, m)$-type formulas for $E$ (which were put forward in the chemical literature between the 1950s and 1990s) revealed that, at least in the case of benzenoid hydrocarbons, none is better than the McClelland approximation (3).

For a long time the McClelland upper bound (2) was the only $(n, m)$-type estimate for $E$, valid for all 
conjugated hydrocarbons. Recently some other generally valid upper and lower bounds for $E$, depending solely on $n$ and/or $m$ were communicated $[9,10]$. On the other hand, quite a few $(n, m)$-type lower and upper bounds applicable only to benzenoid molecules were obtained. These are:

$$
\begin{gathered}
E \leq 2 \sqrt{m+\frac{1}{2} \sqrt{n(n-2)\left(m^{2}-9 m+6 n\right)}}, \\
E \leq 2 \sqrt{\frac{2 m}{n}+\frac{1}{n} \sqrt{\left(9 m n-6 n^{2}-2 m^{2}\right)(n-2)}} \\
+(n-2) \sqrt{\frac{2 m}{n}-\frac{1}{n} \sqrt{\frac{36 m n-24 n^{2}-8 m^{2}}{n-2}}} \\
E \geq \sqrt{4 m^{3} /(9 m-6 n)},
\end{gathered}
$$

and

$$
E \geq \sqrt{3 / 5} \sqrt{2 m n}
$$

The upper bounds (4) and (5) originate from [11] and $[12,13]$, the lower bounds (6) and (7) from [14] and [15], respectively. For lower bounds similar to, but weaker than $(7)$, see $[16,17]$.

Quite recently two more $(n, m)$-type estimates for $E$ were deduced by us [10]:

$$
E \leq \frac{2 m}{n}+\sqrt{(n-1)\left[2 m-\left(\frac{2 m}{n}\right)^{2}\right]}
$$

and

$$
E \leq 2\left(\frac{2 m}{n}\right)+\sqrt{(n-2)\left[2 m-2\left(\frac{2 m}{n}\right)^{2}\right]} .
$$

Inequality (8) is satisfied by all (molecular) graphs whereas (9) is valid for all bipartite (molecular) graphs. Thus, both (8) and (9) hold for benzenoid hydrocarbons.

It is easy to see that the right-hand sides of (8) and (9) are special cases, for $\gamma=1$ and $\gamma=2$, respectively, of an auxiliary function $E^{\star}(\gamma)$, defined as

$$
E^{\star}(\gamma)=\gamma\left(\frac{2 m}{n}\right)+\sqrt{(n-\gamma)\left[2 m-\gamma\left(\frac{2 m}{n}\right)^{2}\right]} .
$$

It is also easy to verify that for $\gamma=0$ the function $E^{\star}(\gamma)$ reduces to the right-hand side of the McClelland inequality (2). These observations motivated us to focus our attention on $E^{\star}(\gamma)$.
In what follows we determine the main analytical properties of $E^{\star}(\gamma)$ and by means of it arrive at novel $(n, m)$-type lower and upper bounds for the total $\pi$ electron energy of benzenoid hydrocarbons, superior to all previously known (above listed) results of this kind. We also obtain novel $(n, m)$-type approximate expressions for $E$, some of which being (slightly) better than the McClelland formula (3).

\section{The Function $E^{\star}(\gamma)$}

In this section it will be assumed that $2 m>n$, a condition which in the case of benzenoid molecules is certainly obeyed. If so, then the $\gamma$-dependence of the function $E^{\star}$ has the form shown in Figure 1.

The function $E^{\star}(\gamma)$ is real-valued for $\gamma<\gamma_{1}$ and $\gamma>\gamma_{2}$, where $\gamma_{1}=n^{2} /(2 m)$ and $\gamma_{2}=n$. In the interval $\left(-\infty, \gamma_{1}\right)$ the function monotonically decreases, whereas in the interval $\left(\gamma_{2},+\infty\right)$ it monotonically increases. Besides,

$$
\begin{aligned}
& \lim _{\gamma \rightarrow-\infty} E^{\star}(\gamma)=\frac{1}{2}(n+2 m), \\
& E^{\star}\left(\gamma_{1}\right)=n, \quad E^{\star}\left(\gamma_{2}\right)=2 m,
\end{aligned}
$$

and

$$
\lim _{\gamma \rightarrow+\infty} \frac{E^{\star}(\gamma)}{\gamma}=2\left(\frac{2 m}{n}\right) .
$$

Note that

$$
\lim _{\gamma \rightarrow-\infty} E^{\star}(\gamma)=\frac{1}{2}\left[E^{\star}\left(\gamma_{1}\right)+E^{\star}\left(\gamma_{2}\right)\right] .
$$

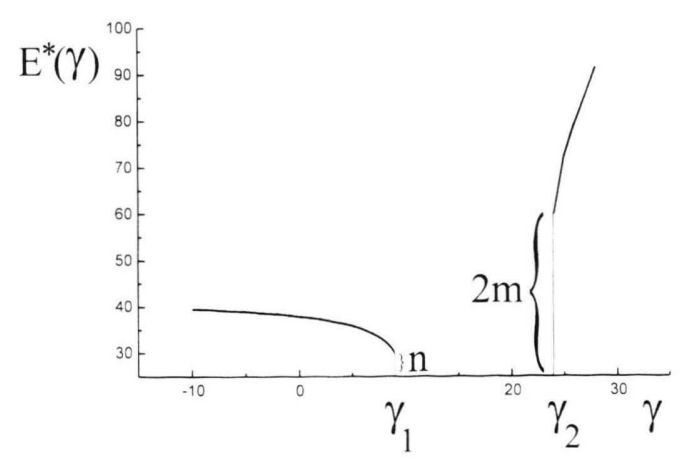

Fig. 1. The $\gamma$-dependence of the function $E^{\star}$, Eq. (10), for typical values of the parameters $n$ and $m ; n=24, m=30$ (coronene); $\gamma_{1}=n^{2} /(2 m)=9.6 ; \gamma_{2}=n=24$. 
From the monotonicity of $E^{\star}$ it follows that

$$
E^{\star}(2)<E^{\star}(1)<E^{\star}(0),
$$

which implies that for all choices of the parameters $n$ and $m$ (such that $2 m>n$ ), the upper bound (9) is better than (8), which, in turn, is better than (2). In fact, any value of $E^{\star}(\gamma)$ for $\gamma<2$ is necessarily an upper bound for $E$.

\section{On the Equation $E^{\star}(\gamma)=E$}

The inequality $E<E^{\star}(\gamma)$ may be obeyed also for some $\gamma>2$. However, for $\gamma=\gamma_{1}$ the inequality $E>$ $E^{\star}(\gamma)$ must hold because the total $\pi$-electron energy of any conjugated molecule is necessarily greater than $n$ [18]. Therefore, in the interval $\left(2, \gamma_{1}\right)$ there exists a value of $\gamma$ (which, in view of the monotonicity of $E^{\star}$, is unique), such that $E^{\star}(\gamma)=E$; we denote it by $\gamma^{\star}$.

Direct calculation gives

$$
\gamma^{\star}=\frac{n\left(2 m n-E^{2}\right)}{2 m(n+2 m-2 E)} .
$$

Hence, the actual value of $\gamma^{\star}$ depends not only on $n$ and $m$, but (through $E$ ) also on the finer details of the molecular structure.

Now, if in any class of conjugated molecules $\gamma_{\text {min }}^{\star}$ and $\gamma_{\max }^{\star}$ are the smallest and greatest values of $\gamma^{\star}$, then, because of the monotonicity of $E^{\star}$,

$$
E^{\star}\left(\gamma_{\max }^{\star}\right) \leq E \leq E^{\star}\left(\gamma_{\min }^{\star}\right)
$$

Recall that $E^{\star}\left(\gamma_{\max }^{\star}\right)$ and $E^{\star}\left(\gamma_{\text {min }}^{\star}\right)$ are certain functions of the variables $n$ and $m$.

In order to learn more on the $\gamma^{\star}$-values of benzenoid hydrocarbons and their structure-dependence, we calculated them for numerous representatives of this class of conjugated compounds. A detailed examination of the results obtained revealed an intriguing property thereof: there exists a good linear correlation between $\gamma^{\star}$ and $n$; moreover, the regression line $\gamma^{\star} \approx a n+b$ has a small, near-zero, $b$-value. (Of course, the values of $a$ and $b$ are sample-dependent.)

An illustrative example is shown in Figure 2.

The results of our numerical studies indicate that the quantity $g=\gamma^{\star} / n$ will vary within rather narrow limits. Indeed, we identified the benzenoid systems with maximal and minimal $g$-value. These are naphthalene $\left(g_{\max }=0.32146 \ldots\right)$ and the (infinite) graphite

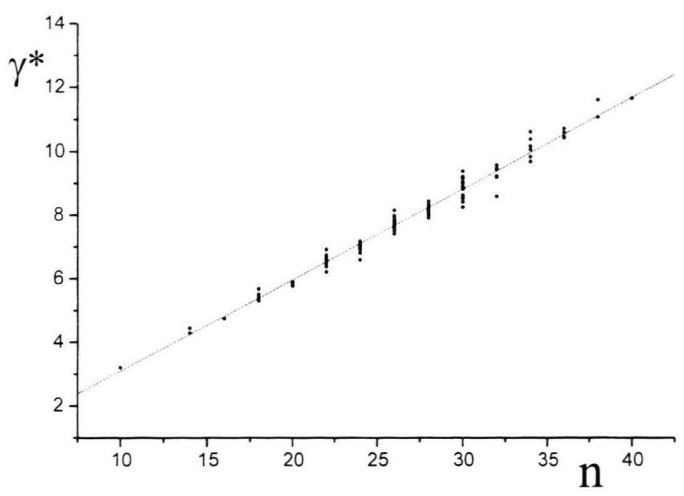

Fig. 2. Correlation between $\gamma^{\star}$ and $n$ for benzenoid hydrocarbons from the book of Zahradnik and Pancir [19] (105 Kekuléan benzenoids) and the respective regression line: $\gamma^{\star}=(0.286 \pm 0.004) n+(0.2 \pm 0.1)$; the correlation coefficient is 0.989 .

lattice $\left(g_{\min }=0.23212 \ldots\right)$. The latter $g$-value was calculated from the data reported in [20].

Thus, as a special case of the relations (12) we get the estimates

$$
E^{\star}\left(n g_{\max }\right) \leq E \leq E^{\star}\left(n g_{\min }\right),
$$

which hold for all benzenoid hydrocarbons and which, in a certain sense, are the best possible. Somewhat weaker, but algebraically more appealing bounds for the total $\pi$-electron energy of benzenoid systems are obtained when in (13) the values $1 / 3$ and $1 / 5$ are substituted instead of $g_{\max }$ and $g_{\min }$, respectively. This results in

$$
\begin{aligned}
& \frac{2}{3}\left(m+\sqrt{3 m n-2 m^{2}}\right) \\
& <E<\frac{2}{5}\left(m+\sqrt{10 m n-4 m^{2}}\right) .
\end{aligned}
$$

In the left part of Table 1 we compare the lower bounds (6), (7) and (14) for a few chemically relevant values of $n$ and for all possible [21,22] values of $m$ that can occur in benzenoid hydrocarbons. It is seen that the new estimate (14) is far superior to any of the previously reported ones.

In the right part of Table 1 we compare the upper bounds (2), (4), (5), (8), (9) and (14) for the same choice of the parameters $n$ and $m$. For $n>22$ the new estimate (14) is better than any of the previously reported ones. For $n \leq 22$ (that is for just a few benzenoid systems) the Cioslowski inequality (5) [12, $13]$ is better than ours. 
Table 1. Bounds for the total $\pi$-electron energy of benzenoid hydrocarbons $C_{n} H_{3 n-2 m}$; for details see text.

\begin{tabular}{|c|c|c|c|c|c|c|c|c|c|c|}
\hline \multirow[b]{2}{*}{$\mathrm{n}$} & \multicolumn{4}{|c|}{ — Lower bonds - } & \multicolumn{6}{|c|}{ - Upper bonds - } \\
\hline & $\mathrm{m}$ & Eq. (6) & Eq. (7) & Eq. (14) & Eq. (2) & Eq. (4) & Eq. (5) & Eq. (8) & Eq. (9) & Eq. (14) \\
\hline 10 & 11 & 11.6839 & 11.4891 & 13.5872 & 14.8324 & 14.3523 & 14.0100 & 14.6274 & 14.3277 & 14.3277 \\
\hline 14 & 16 & 16.5247 & 16.3951 & 19.0994 & 21.1660 & 20.6621 & 20.0887 & 20.9427 & 20.6529 & 20.3485 \\
\hline 16 & 19 & 19.1262 & 19.0997 & 21.8560 & 24.6577 & 24.1292 & 23.4266 & 24.4066 & 24.0907 & 23.5800 \\
\hline 18 & 21 & 21.3854 & 21.2979 & 24.5830 & 27.4955 & 26.9812 & 26.1937 & 27.2621 & 26.9776 & 26.3600 \\
\hline 20 & 24 & 24.0000 & 24.0000 & 27.3137 & 30.9839 & 30.4530 & 29.5446 & 30.7295 & 30.4250 & 29.5840 \\
\hline 21 & 25 & 25.1259 & 25.0998 & 28.6852 & 32.4037 & 31.8785 & 30.9298 & 32.1571 & 31.8651 & 30.9762 \\
\hline 22 & 26 & 26.2537 & 26.1992 & 30.0525 & 33.8231 & 33.3033 & 32.3153 & 33.5835 & 33.3022 & 32.3672 \\
\hline 22 & 27 & 26.6326 & 26.6983 & 30.0000 & 34.4674 & 33.9252 & 32.9018 & 34.1954 & 33.8747 & 32.7964 \\
\hline 23 & 28 & 27.7533 & 27.7993 & 31.3859 & 35.8887 & 35.3516 & 34.2884 & 35.6248 & 35.3165 & 34.1922 \\
\hline 24 & 29 & 28.8758 & 28.8998 & 32.7663 & 37.3095 & 36.7772 & 35.6751 & 37.0530 & 36.7555 & 35.5867 \\
\hline 24 & 30 & 29.2770 & 29.3939 & 32.6491 & 37.9473 & 37.3972 & 36.2636 & 37.6603 & 37.3265 & 36.0000 \\
\hline 26 & 31 & 31.1258 & 31.0998 & 35.5140 & 40.1497 & 39.6264 & 38.4492 & 39.9058 & 39.6268 & 38.3723 \\
\hline 26 & 32 & 31.5114 & 31.5975 & 35.4440 & 40.7922 & 40.2508 & 39.0394 & 40.5210 & 40.2099 & 38.7969 \\
\hline 30 & 36 & 36.0000 & 36.0000 & 40.9706 & 46.4758 & 45.9501 & 44.5929 & 46.2288 & 45.9514 & 44.3760 \\
\hline 30 & 37 & 36.3904 & 36.4966 & 40.8874 & 47.1169 & 46.5760 & 45.1858 & 46.8463 & 46.5419 & 44.7973 \\
\hline 30 & 38 & 36.8085 & 36.9865 & 40.7101 & 47.7493 & 47.1959 & 45.7776 & 47.4542 & 47.1214 & 45.1973 \\
\hline 34 & 41 & 40.8756 & 40.8999 & 46.4238 & 52.8015 & 52.2741 & 50.7447 & 52.5521 & 52.2760 & 50.3788 \\
\hline 34 & 42 & 41.2695 & 41.3957 & 46.3303 & 53.4416 & 52.9011 & 51.3397 & 53.1714 & 52.8719 & 50.7976 \\
\hline 34 & 43 & 41.6876 & 41.8856 & 46.1532 & 54.0740 & 53.5225 & 51.9333 & 53.7824 & 53.4585 & 51.1976 \\
\hline 40 & 49 & 48.3867 & 48.4974 & 54.5553 & 62.6099 & 62.0751 & 60.2838 & 62.3491 & 62.0648 & 59.5920 \\
\hline 40 & 50 & 48.7950 & 48.9898 & 54.4152 & 63.2456 & 62.7007 & 60.8805 & 62.9669 & 62.6628 & 60.0000 \\
\hline 40 & 51 & 49.2224 & 49.4773 & 54.1990 & 63.8749 & 63.3214 & 61.4755 & 63.5779 & 63.2533 & 60.3920 \\
\hline 40 & 52 & 49.6670 & 49.9600 & 53.8963 & 64.4981 & 63.9371 & 62.0687 & 64.1821 & 63.8365 & 60.7680 \\
\hline 50 & 61 & 60.3844 & 60.4979 & 68.2186 & 78.1025 & 77.5714 & 75.4123 & 77.8474 & 77.5743 & 74.3856 \\
\hline 50 & 62 & 60.7866 & 60.9918 & 68.0998 & 78.7401 & 78.2006 & 76.0126 & 78.4710 & 78.1827 & 74.7984 \\
\hline 50 & 63 & 61.2048 & 61.4817 & 67.9230 & 79.3725 & 78.8256 & 76.6114 & 79.0891 & 78.7852 & 75.1984 \\
\hline 50 & 64 & 61.6376 & 61.9677 & 67.6822 & 80.0000 & 79.4465 & 77.2084 & 79.7019 & 79.3821 & 75.5856 \\
\hline 50 & 65 & 62.0837 & 62.4500 & 67.3703 & 80.6226 & 80.0632 & 77.8036 & 80.3095 & 79.9733 & 75.9600 \\
\hline 50 & 66 & 62.5421 & 62.9285 & 66.9783 & 81.2404 & 80.6760 & 78.3969 & 80.9119 & 80.5589 & 76.3215 \\
\hline 60 & 74 & 72.7808 & 72.9932 & 81.7747 & 94.2338 & 93.6979 & 91.1675 & 93.9709 & 93.6926 & 89.5947 \\
\hline 60 & 75 & 73.1925 & 73.4847 & 81.6228 & 94.8683 & 94.3260 & 91.7692 & 94.5937 & 94.3029 & 90.0000 \\
\hline 60 & 76 & 73.6170 & 73.9730 & 81.4202 & 95.4987 & 94.9505 & 92.3693 & 95.2121 & 94.9084 & 90.3947 \\
\hline 60 & 77 & 74.0532 & 74.4580 & 81.1625 & 96.1249 & 95.5714 & 92.9678 & 95.8261 & 95.5093 & 90.7787 \\
\hline 60 & 78 & 74.5004 & 74.9400 & 80.8444 & 96.7471 & 96.1888 & 93.5645 & 96.4358 & 96.1057 & 91.1520 \\
\hline 60 & 79 & 74.9578 & 75.4188 & 80.4596 & 97.3653 & 96.8027 & 94.1594 & 97.0413 & 96.6975 & 91.5146 \\
\hline 60 & 80 & 75.4247 & 75.8947 & 80.0000 & 97.9796 & 97.4132 & 94.7526 & 97.6427 & 97.2850 & 91.8665 \\
\hline
\end{tabular}

\section{New $(n, m)$-Type Approximate Formulas for $E$}

The main result communicated in this section is the novel $(n, m)$-type approximation (17) for the total $\pi$-electron energy of benzenoid hydrocarbons.

We have already seen that for each conjugated molecule there is a value of $\gamma$ for which $E^{\star}(\gamma)=E$. This suggests that for a given class of conjugated molecules one may find a $\gamma$ which (on average) optimizes the approximation

$$
E^{\star}(\gamma) \approx E
$$

for all members of the class.
For a representative (frequently employed [3]) set of 105 polycyclic Kekuléan benzenoid hydrocarbons from the book of Zahradnik and Pancir [19], we found that the choice $\gamma=0.294$ minimizes the average relative error of (15). The minimum of this error is surprisingly sharp: whereas for $\gamma=0.294$ it is $0.49 \%$, for $\gamma=0.284$ and $\gamma=0.304$ it increases to $0.81 \%$ and $0.62 \%$, respectively.

In view of the success of the McClelland approximation (3), which is equal to $A E^{\star}(0)$, it is purposeful to examine the formula $A E^{\star}(\gamma)$ as an extended, and possibly better approximation for total $\pi$-electron energy. This formula can be optimized with respect to both $A$ and $\gamma$. 


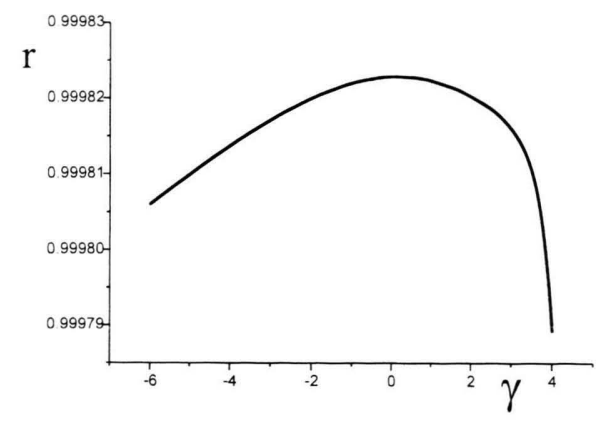

Fig. 3. The correlation coefficient $r$ of the approximation (16), calculated for the Zahradnik-Pancir data set [19]; the maximum $(r=0.999823)$ is attained at $\gamma=+0.1$, which practically coincides with the McClelland approximation (3).

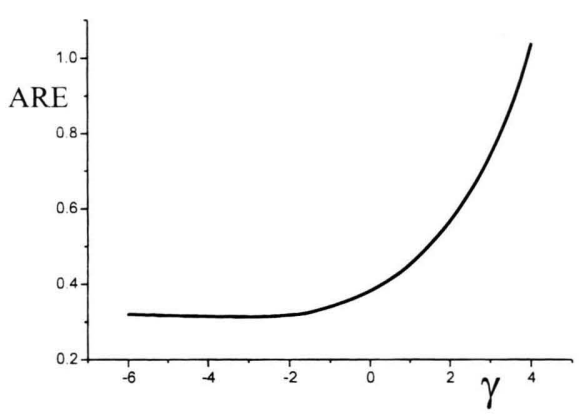

Fig. 4. The average relative error ARE (in \%) of the approximation (5), calculated for the Zahradnik-Pancir data set [19]; the (very shallow) minimum $(\mathrm{ARE}=0.31 \%)$ is attained at $\gamma=-2.9$, significantly far from, and significantly better than the McClelland approximation (3).

The quality of the approximation (16)

$$
E \approx A E^{\star}(\gamma)
$$

was tested using the above described standard data base [19]. The main results obtained are shown in Figs. 3 and 4.

[1] L. J. Schaad and B. A. Hess, J. Amer. Chem. Soc. 94, 3068 (1972).

[2] I. Gutman and O. E. Polansky, Mathematical Concepts in Organic Chemistry, Springer-Verlag, Berlin 1986.

[3] I. Gutman, Topics Curr. Chem. 162, 29 (1992).

[4] G. G. Hall, Proc. Roy. Soc. London A 229, 251 (1955).

[5] G. G. Hall, Trans. Faraday Soc. 53, 573 (1957).

[6] B. J. McClelland, J. Chem. Phys. 54, 640 (1971).
An intriguing finding is that the McClelland formula (3) has the highest correlation coefficient among all approximate expressions of the type (16). This corroborates the earlier conclusion $[3,7,8,16,23]$ that the $(n, m)$-dependence of the total $\pi$-electron energy of benzenoid hydrocarbons is well represented by the function $\sqrt{2 m n}$. This, however, does not require that the McClelland approximation be the most accurate one in the class of formulas (16) considered.

Indeed, from a practical point of view the best approximate formula is the one having the smallest average relative error, $A R E$. Without losing accuracy we may set $\gamma=-3$ instead of the optimal value $\gamma=-2.9$, cf. Figure 4 . Whereas the $A R E$ of the formula (16) for $\gamma=-3$ is $0.313 \%$, the McClelland formula $(\gamma=0)$ has $A R E=0.382 \%$. Thus the new approximation

$$
E \approx A E^{\star}(-3)=A\left[\sqrt{(n+3)\left(2 m+\frac{12 m^{2}}{n^{2}}\right)}-\frac{6 m}{n}\right]
$$

with $A=0.896 \pm 0.003$ is (on average) by some $22 \%$ more accurate than the McClelland formula. Within the Zahradnik-Pancir sample [19] the maximal observed error of formula (17) is $1.0 \%$. The respective correlation coefficient is $r=0.999817$, insignificantly differing from the maximal value $r=0.999823$ at $\gamma=0$.

From Fig. 4 is seen that the accuracy of the approximation (16) (as measured by the average relative error) rapidly improves as $\gamma$ decreases, but for $\gamma<-2$ becomes almost constant. This is a direct consequence of the property (11) of the function $E^{\star}(\gamma)$, cf. Figure 1.

In summary, the ( $n, m)$-type formula (17) is somewhat (by some 20\%) more accurate than the classical McClelland formula. It is capable of reproducing (on average) some $99.7 \%$ of the total $\pi$-electron energy.

[7] I. Gutman, L. Nedeljković, and A. V. Teodorović, J. Serb. Chem. Soc. 48, 495 (1983).

[8] I. Gutman, S. Marković, A. V. Teodorović, and Ž. Bugarčić, J. Serb. Chem. Soc. 51, 145 (1986).

[9] G. Caporossi, D. Cvetković, I. Gutman, and P. Hansen, J. Chem. Inf. Comput. Sci. 39, 984 (1999).

[10] J. H. Koolen, V. Moulton, and I. Gutman, Chem. Phys. Letters, in press. 
[11] L. Türker, Commun. Math. Chem. (MATCH) 16, 83 (1984).

[12] J. Cioslowski, Z. Naturforsch. 40a, 1167 (1985).

[13] J. Cioslowski and I. Gutman, Z. Naturforsch. 41a, 861 (1986).

[14] J. Cioslowski, Int. J. Quantum Chem. 34, 217 (1988).

[15] D. Babić and I. Gutman, Commun. Math. Chem. (MATCH) 32, 7 (1995).

[16] I. Gutman, J. Chem. Soc. Faraday Trans. 86, 3373 (1990).

[17] L. Türker, Commun. Math. Chem. (MATCH) 30, 243 (1994).
[18] W. England and K. Ruedenberg, J. Amer. Chem. Soc. 95, 8769 (1973).

[19] R. Zahradnik and J. Pancir, HMO Energy Characteristics, Plenum Press, New York 1970.

[20] I. Gutman, S. J. Cyvin, and V. Ivanov-Petrović, Z. Naturforsch. 53a, 699 (1998).

[21] J. R. Dias, Z. Naturforsch. 44a, 765 (1989).

[22] J. Brunvoll and S. J. Cyvin, Z. Naturforsch. 45a, 69 (1990).

[23] J. Cioslowski and O. E. Polansky, Theor. Chim. Acta 74, 55 (1988). 